\title{
Melhorando a Colaboração de um Ambiente Virtual de Aprendizagem usando um Agente Pedagógico Animado 3D
}

\author{
Márcio Aurélio dos Santos Alencar, José Francisco de Magalhães Netto
}
Programa de Pós-Graduação em Informática - Instituto de Computação - ICOMP - Universidade Federal do Amazonas (UFAM)

Av. Gen. Rodrigo Otávio, 6.200, Setor Norte do Campus Universitário - Coroado I 69077-000 - Manaus - AM - Brasil

marcio.alencar@icomp.ufam.edu.br, jnetto@icomp.ufam.edu.br

\begin{abstract}
This paper describes the architecture and operation of the $3 D$ animated pedagogical agent TUCUMÃ, integrated to a Virtual Learning Environment, using Multi-Agent System, AIML, Affective Computing and Sentiment analysis. A partial evaluation of the project was carried out with 5 classes of the training course of a distance education school, in order to Sentiment analysis of the texts published in the forums and chats of the VLE. The results are promising, highlighting the importance of the approach.
\end{abstract}

Resumo. Este artigo descreve a arquitetura e funcionamento do agente animado pedagógico 3D TUCUMÃ, integrado a um Ambiente Virtual de Aprendizagem, usando Sistema Multiagente, AIML, Computação Afetiva e Análise de Sentimentos. Uma avaliação parcial do projeto foi realizada com 5 turmas do curso de formação de tutores de uma escola de Educação a Distância com objetivo de analisar o sentimento dos textos publicados nos fóruns e chats do AVA. Os resultados são promissores, evidenciando a importância da abordagem.

\section{Introdução}

Cresce o número de pesquisas com objetivo de realizar o acompanhamento de alunos em Ambientes Virtuais de Aprendizagem (AVA), tendo em vista as limitações desses ambientes, dificultando o trabalho dos tutores em acompanhar as atividades dos alunos. Outro problema frequente que observamos é o grande número de mensagens postadas pelos alunos em fóruns e a falta de feedback dos tutores.

Em um AVA temos várias ferramentas essenciais para a construção coletiva do conhecimento, dentre elas podemos destacar o fórum e o chat, que possibilitam ao participante fazer reflexões, organizar ideias e se aprofundar em tópicos de um curso (Alencar e Netto, 2011a). Por meio dessas ferramentas os alunos podem expressar seus sentimentos e opiniões.

A metodologia adotada neste trabalho iniciou com uma revisão bibliográfica e sistemática sobre computação afetiva, análise de sentimentos e Agente Pedagógico Animado, para entender sua atuação, quais tecnologias são usadas na implementação e suas contribuições para educação.

Com base nesses estudos, o presente artigo propõe uma abordagem para a solução desse problema baseada no desenvolvimento de um Agente Pedagógico Animado (APA) 3D integrado a um AVA, utilizando Sistema Multiagente (SMA), AIML (Artificial Intelligence Markup Language), Computação Afetiva e Análise de Sentimentos.

O Agente Pedagógico Animado TUCUMÃ (TUtor Collaborator Using Multi-Agent system) é responsável pelo acompanhamento das atividades do aluno no curso, por responder 
VI Congresso Brasileiro de Informática na Educação (CBIE 2017)

Anais do XXVIII Simpósio Brasileiro de Informática na Educação (SBIE 2017)

suas dúvidas, além de informar quais atividades estão pendentes. A interação do aluno com o APA é bem dinâmica, pois com sua interface amigável, é possível ouvir sua voz e visualizar seus movimentos.

Para descrever a pesquisa, este artigo está organizado da seguinte forma: a seção 2 apresenta os trabalhos relacionados aos assuntos: Agentes Pedagógicos Animados, Computação Afetiva e Análise de Sentimentos, além de nossas contribuições; a seção 3 apresenta a arquitetura; a seção 4 explica o funcionamento do projeto; a seção 5 descreve o experimento; o artigo é finalizado com a seção 6 , onde estão as conclusões e os trabalhos futuros.

\section{Trabalhos Relacionados e Contribuições}

\subsection{Agentes Pedagógicos Animados}

Os Agentes Pedagógicos Animados (APA) são agentes educacionais que são representados por um personagem animado que interage com alunos. Eles usam recursos de multimídia para oferecer ao usuário uma imagem mais próxima a humana.

Esses agentes educacionais se comunicam por meio de gestos e expressões faciais, colaborando com o tutor no acompanhamento dos estudantes. O trabalho de (Martins et al., 2014) destaca a importância do emprego de gestos por APA em ambientes educacionais. Em sua pesquisa foram avaliados vídeos de professores ensinado Matemática, onde observou-se seus gestos e definido um parâmetro aproximado de gestos usado pelos professores. Esses gestos foram usados no APA integrado ao PAT2Math.

O trabalho de [Fontes et al., 2012] desenvolveu o APA integrado em um AVA, com o papel de tutor, auxiliando os estudantes na aquisição de conhecimento, procurando identificar estudantes com comportamento passivo, motivando o estudante a participar das atividades e discussões, além de recomendar materiais educacionais que possam sanar as dúvidas dos estudantes durante o processo de aplicação da PBL( (Problem-Based Learning).

Os pesquisadores [Mateus et al, 2014] utilizaram um APA para acompanhar e monitorar estudantes do curso de Engenharia na disciplina de Estatística em um AVA. O APA interage com os estudantes por meio de mensagens, alertas e elogios com proposito de redirecionar os estudantes para a atividade mais adequada ao seu desempenho.

Greta (Niewiadomski et al., 2009) é um agente animado tridimensional, usando padrão de animação compatível com MPEG-4, que se comunica com estudantes usando uma rica paleta de comportamentos verbais e não-verbais. Greta tem a capacidade de expressar emoções por meio de expressões faciais, gestos, olhar, fala e movimentos de cabeça.

O agente pedagógico Dóris 3D desenvolvido por (Frozza et al., 2009), inserido em um AVA, expressa emoções (alegria, tristeza, dúvida, atenção, surpresa, indignação e expectativa), a fim de facilitar o aprendizado de estudantes que interagem com o sistema. $\mathrm{O}$ agente possui os seguintes módulos: perceptivo, cognitivo e reativo.

Sansonnet et al (2012) desenvolveram o framework Divalite, que permite a criação de Agentes Pedagógicos Animados para ambientes Web, onde é possível configurar os movimentos do APA por meio de implementação nas linguagens HTML, JavaScript e Jquery. Esse framework é gratuito para uso em pesquisas e softwares educacionais.

A pesquisa de Graesser et al., (2012), descreveu o AutoTutor, um agente 3D que ajuda estudantes a aprender a Física Newtoniana e conhecimentos de informática através do diálogo em linguagem natural. Este projeto verifica as emoções e conhecimentos do aluno por meio de diálogos, expressões faciais, movimentos do corpo e entonação de voz, facilitando a construção do conhecimento interativo. 
O MAXINE é uma ferramenta que permite o gerenciamento de Agentes Pedagógicos Animados, possibilitando interação multimodal e emocional com o usuário. $\mathrm{O}$ motor pode reconhecer o estado emocional do usuário e utilizar um agente virtual para expressar emoções por meio de posturas corporais e gestos. O MAXINE já foi aplicado em vários ambientes, usando agentes virtuais diferentes. Como agente jovem, ajudando crianças com necessidade especial, como mordomo, controlando uma casa remotamente, e como um tutor, ajudando estudantes universitários no aprendizado de computação gráfica (Baldassarri e Cerezo, 2012).

Guru é um sistema de tutoria inteligente, que ajuda estudantes do ensino médio no aprendizado de Biologia. O agente transfere seu conhecimento de Biologia para os alunos por meio de um painel multimídia que apresenta filmes, diagramas interativos e mídias instrucionais. Assim como acontece na tutoria humana, Guru pode avaliar precisamente as respostas dos estudantes (Olney et al., 2012).

\subsection{Computação Afetiva}

É notável um aumento no número de pesquisas aplicadas em Informática na Educação, usando Computação Afetiva, principalmente no uso de Agentes Pedagógicos Animados. Segundo Picard (2003), Computação Afetiva (CoA) é a área da informática que busca reconhecer e representar a afetividade na interação homem-máquina, ou seja, o uso das emoções em diferentes aspectos nos sistemas computacionais.

Pesquisas voltadas para a Informática na Educação usando CoA, tem por objetivo perceber, identificar e medir fenômenos afetivos de estudantes por meios tecnológicos, e exibir emoções na interação entre o agente pedagógico animado e o estudante. Conforme Picard (2003), a máquina deve saber identificar o estado afetivo do estudante, entender e agir no estado afetivo usando técnicas de reconhecimento de padrões, processamento de som e imagens, além do emprego de algoritmos computacionais para avaliar e responder aos estados afetivos.

Alunos utilizando fóruns, chats e mensagens, têm a oportunidade de interagir com o tutor, trabalhando de forma colaborativa, compartilhando informação, opiniões e sentimentos. Por meio dessas interações síncronas e assíncronas, o tutor pode estimular as relações socioafetivas, como: motivar, encorajar e apoiar alunos por meio de palavras.

Assim como ocorre relações afetivas entre tutor/aluno em cursos presenciais, por meio de gestos e falas, em cursos de Educação a Distância (EaD), é necessário que ocorra também esse vínculo e participações pois ajudam na permanência do aluno no curso, além de apoiar em momentos de dificuldades na realização de atividades.

A presença social (PS) de alunos em AVA é fundamental para o aprendizado, tendo em vista que em cursos $\mathrm{EaD}$ não se tem contato direto com os alunos, com isso podemos destacar um crescimento no número de pesquisas que usam agentes pedagógicos animados, que substituem a presença do tutor humano (Bastos et al., 2011).

$\mathrm{O}$ vínculo afetivo entre tutor e aluno tem papel importante na mediação pedagógica, pois os alunos esperam do tutor atenção, motivação e incentivo nos momentos virtuais e presenciais, isto é, os alunos se sentem mais acolhidos e seguros quando há essa relação afetiva e cognitiva na realização das atividades (Cruz e Souza, 2014).

\subsection{Análise de Sentimentos}

Avaliar as opiniões e os sentimentos dos estudantes em Ambientes Virtuais de Aprendizagem, ajuda o tutor a analisar seus métodos de ensino, podendo identificar pontos positivos e negativos, auxiliando na tomada de decisões, além de estimular estudantes no aprendizado. 
Assim como existe emoção na comunicação entre pessoas, podemos identificar também esse sentimento entre homem e computador.

Dentre as diversas pesquisas realizadas na Computação Afetiva, podemos destacar a identificação automática de emoções em informação textual, conhecida como Análise de Sentimentos (AS). Análise de sentimentos ou Mineração de Opinião é um campo de estudo que analisa opiniões, sentimentos, avaliações, atitudes e emoções expressas em diferentes mídias sociais (Liu, 2015).

Muitas pesquisas vêm sendo desenvolvidas para criar métodos computacionais que sejam capazes de identificar emoções em textos, contudo grande parte dessas pesquisas usam textos na língua Inglesa (Cambria et al., 2016), focando principalmente na identificação da polaridade das emoções nos textos, ou seja, buscando identificar se os textos são positivos ou negativos.

Machado et al., 2015 implementaram o Personalitatem Lexicon, composto de lexemas de conotação afetiva baseada nos traços de personalidade, que ajuda a identificar os traços de personalidade de estudantes, seu trabalho analisou textos de chats de uma disciplina de um curso.

Segundo [Longhi et al., 2009] um dos desafios da Inteligência Artificial é tratar computacionalmente sentimentos, opiniões e a subjetividade em texto. Nesse projeto os pesquisadores procuram identificar os estados de ânimo (Satisfeito, Insatisfeito, Animado ou Desanimado) nos textos postados por alunos no fórum de um AVA.

\subsection{Contribuições}

Acreditamos que as contribuições do nosso trabalho em relação aos demais é que nosso Agente Pedagógico Animado utiliza Sistema Multiagente (SMA), AIML, Computação Afetiva e Análise de Sentimentos para acompanhar as atividades e interagir com os estudantes de um curso EaD. O SMA é responsável pelo acompanhamento das atividades, o AIML usado para compor a base de conhecimentos utilizada para dialogar com os alunos e a Computação Afetiva, juntamente com a Análise de Sentimentos são usadas para verificar o estado emocional dos alunos.

\section{Arquitetura}

No trabalho de Alencar e Netto (2014) podemos observar uma arquitetura que serviu de base para a atual arquitetura do APA TUCUMÃ (Figura 1), composta pelos principais elementos: Estudante, Tutor, Agente Pedagógico Animado, sintetizador de voz, sistema Web, base de conhecimento, Interpretador AIML, Interpretador de sentimentos, agentes inteligentes responsáveis pelas atividades, pelos sentimentos e pelos gestos do APA. 
VI Congresso Brasileiro de Informática na Educação (CBIE 2017)

Anais do XXVIII Simpósio Brasileiro de Informática na Educação (SBIE 2017)

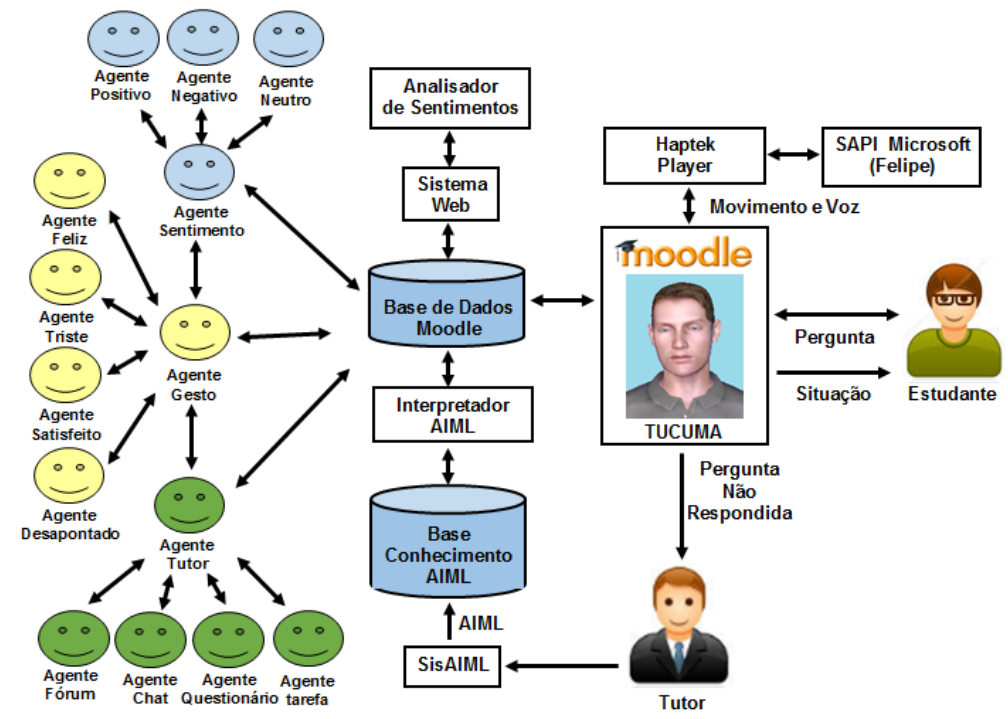

Figura 1. Arquitetura do APA TUCUMÃ

O protótipo do avatar foi desenvolvido usando as tecnologias: LAMP (Linux,Apache,MySQL,PHP), AIML (Artificial Intelligence Markup Language), Intepretador AIML Program-O, Framework JADE (Java Agent DEvelopment Framework), Moodle (Modular Object-Oriented Dynamic Learning Environment), Sintetizador de voz, Haptek Player e Sentic Computing.

\section{Funcionamento}

O agente Pedagógico Animado TUCUMÃ está inserido no AVA da Escola de Educação Profissional a Distância - CETAM EaD com objetivo de auxiliar alunos no acompanhamento das atividades, estimular o aprendizado, sanar dúvidas, além de ajudar tutores em suas atividades cotidianas.

Para criar a base de conhecimento do TUCUMÃ, foi desenvolvido o sistema web SisAIML. Esse sistema está integrado ao AVA, possibilitado ao tutor responsável pelo curso ou disciplina, logar no SisAIML com a mesma conta do AVA. Antes de um curso iniciar no AVA, o tutor a distância cadastra as perguntas e respostas referentes ao curso, com isso o sistema se encarrega de elaborar uma variedade de formas do estudante perguntar, e por fim o sistema gera os arquivos AIML, que serão usados pelo interpretador AIML.

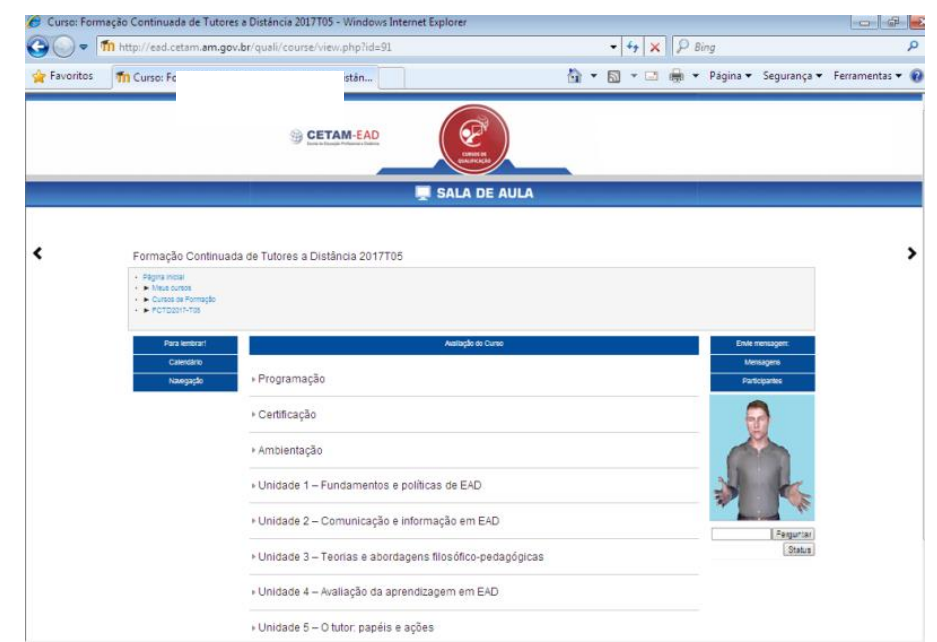

Figura 2. Agente Pedagógico Animado 3D integrado ao AVA 
O estudante ao acessar o curso (http://ead.cetam.am.gov.br/quali/), pode interagir com o APA (Figura 2), que está no lado direito da interface, apresentando-se de forma gráfica dinâmica, por meio de comportamentos físicos e verbais, capaz de expressar emoções que irão ajudar no aprendizado, de forma a parecer um tutor humano.

Para o estudante sanar dúvidas sobre a disciplina ou saber sua situação no curso, basta interagir com o avatar TUCUMÃ. Caso o estudante faça uma pergunta ao avatar TUCUMÃ, serão acionados os agentes inteligentes responsáveis pelas atividades que com a ajuda do interpretador AIML irá consultar sua base de conhecimento para retornar a resposta. Para apresentar os gestos usados pelo avatar para interagir com o estudante são acionados os agentes inteligentes responsáveis pelos gestos.

Se o estudante deseja saber sua situação no curso, irá clicar no botão STATUS, com isso o avatar irá consultar os agentes inteligentes responsáveis pelas atividades do AVA. Para isso, esses agentes acessam as informações sobre a utilização das principais ferramentas do Moodle: Fórum, chat, questionário e tarefa. Eles irão analisar se as atividades foram finalizadas dentro do prazo.

Para avaliar o estado emocional do estudante, os agentes inteligentes responsáveis pelos sentimentos contam com a ajuda do interpretador de sentimentos, que é encarregado de extrair os sentimentos dos textos postados no fórum e chat pelo estudante. Para falar com o estudante o avatar utiliza um sintetizador de voz no idioma Português do Brasil, na voz Felipe.

Durante a interação com o estudante, o avatar TUCUMÃ pode expressar várias emoções: quando o aluno está em dia com suas atividades, o agente sorri, mostrando estar feliz (Figura 3a), quando o aluno tem atividades atrasadas, o agente chama atenção do estudante e fica triste (Figura $3 \mathrm{~b}$ ), quando o aluno tem dúvidas sobre um assunto do curso, o avatar fica satisfeito e abre os braços para explicar (Figura 3c) e quando o avatar não sabe a resposta, fica desapontado, coloca a mão na testa e encaminha a pergunta ao tutor (Figura 3d).

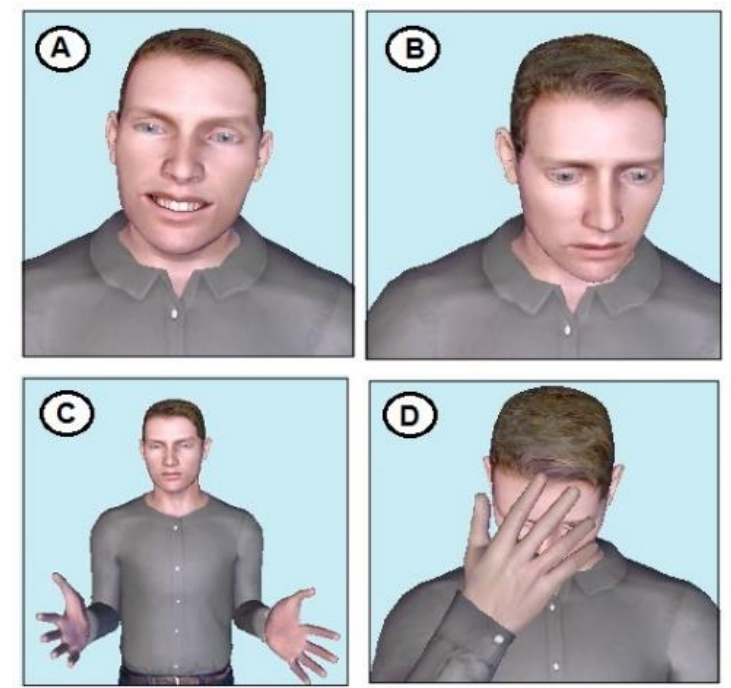

Figura 3. Emoções do Agente Pedagógico Animado

Segundo (Jaques e Nunes, 2012) existem quatro modos de reconhecimento das emoções pelo usuário: pela voz, pelas ações do usuário no ambiente, expressões faciais e sinais fisiológicos (batimento cardíaco, respiração, etc). A emoção é transmitida através de comportamentos físicos e verbais: a animação do agente representa o físico e a fala, o verbal. As pesquisas de (Ekman, 2011) buscam representar as emoções universais, e classificou em sete emoções: nojo, raiva, medo, tristeza, alegria, surpresa e desprezo. 
Para definir os gestos e emoções usadas na interação do APA TUCUMÃ utilizamos o software HapFACS, apresentado por (Amini et al., 2015), que usa a tecnologia Haptek Player para visualizar Agentes Pedagógicos Animados 3D. Com o HapFACS é possível retratar, por meio de gestos e expressões faciais, todas essas emoções propostas por (Ekman, 2011).

Podemos destacar também a importância da integração de várias tecnologias em prol da educação, como vemos na pesquisa de [Clavel et al., 2016] vemos a integração de várias tecnologias em prol da educação, onde temos um APA 3D, destacando o uso de gestos e voz, juntamente com o uso da análise de sentimentos. Assim como [Alencar e Netto, 2011b] realizaram experimentos utilizando Sistema Multiagente e AIML para acompanhar atividades produzidas por estudantes de um curso Ead.

\section{Experimento}

O experimento foi realizado utilizando dados reais de 5 (cinco) turmas do curso de formação de tutores da Escola de Educação Profissional a Distância - CETAM EaD com média de 40 alunos por turma e com carga horária de 100 horas, que foi realizado no período de maio a julho de 2017. Cada curso teve 5 (cinco) módulos, sendo que o fórum e o chat foram as principais atividades realizadas pelos alunos.

Como forma de avaliar parcialmente o projeto, foram analisados os sentimentos das mensagens postadas pelos alunos nos fóruns e chats dos cursos, seguindo 3 etapas: coleta de dados, classificação e sumarização, conforme Figura 4.

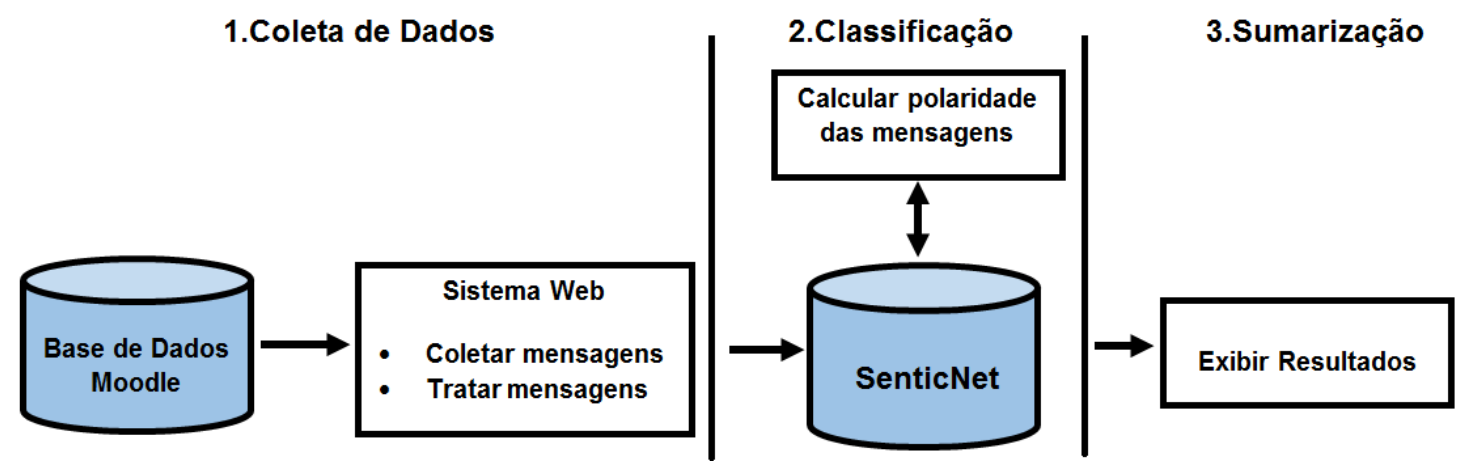

Figura 4. Etapas da Análise de Sentimentos

Para coletar dos dados foi desenvolvido um sistema web na linguagem PHP, que coleta as mensagens postadas pelos alunos nos fóruns e chats dos cursos, remove os marcadores HTML das mensagens, remove as stopwords e divide a mensagem em palavras.

$\mathrm{Na}$ etapa de classificação das mensagens foi utilizado a SenticNet4 (http://sentic.net/), uma ferramenta que realiza mineração de opinião, análise de sentimentos e explora técnicas de Inteligência Artificial e Web Semântica (Cambria et al., 2016). Essa ferramenta possui uma base de conhecimento composta por 50.000 conceitos, disponível em 40 idiomas, onde cada conceito possui uma polaridade (positiva, negativa e neutra), uma emoção (felicidade, admiração, interesse, surpresa, tristeza, nojo, raiva, medo), está associada a um grupo de quatro dimensões: simpatia, atenção, sensibilidade e aptidão, do modelo Hourglass emotions.

A ferramenta SenticNet verificou a polaridade e sentimento de cada palavra contida em cada mensagem, e para definir o sentimento e polaridade da mensagem contabilizamos o sentimento que mais se destacava.

$\mathrm{Na}$ etapa de sumarização podemos exibir para cada mensagem (fórum e chat), o valor de sua polaridade, assim como a emoção do texto do aluno.

$\mathrm{Na}$ etapa da coleta dos dados, verificamos que os alunos das cinco turmas fizeram 7536 posts nos fóruns (6721 positivos, 483 negativos e 332 neutros) e 4747 posts nos chats 
VI Congresso Brasileiro de Informática na Educação (CBIE 2017)

Anais do XXVIII Simpósio Brasileiro de Informática na Educação (SBIE 2017)

(3066 positivos, 712 negativos e 966 neutros). No processo de classificação contabilizamos as polaridades e emoção para cada mensagem. Na etapa de sumarização consolidamos os resultados por turma (T1,T2,T3,T4,T5), que são apresentados nos Gráficos 1 e 2.

\section{Gráfico 1 - Emoções identificadas nos fóruns}

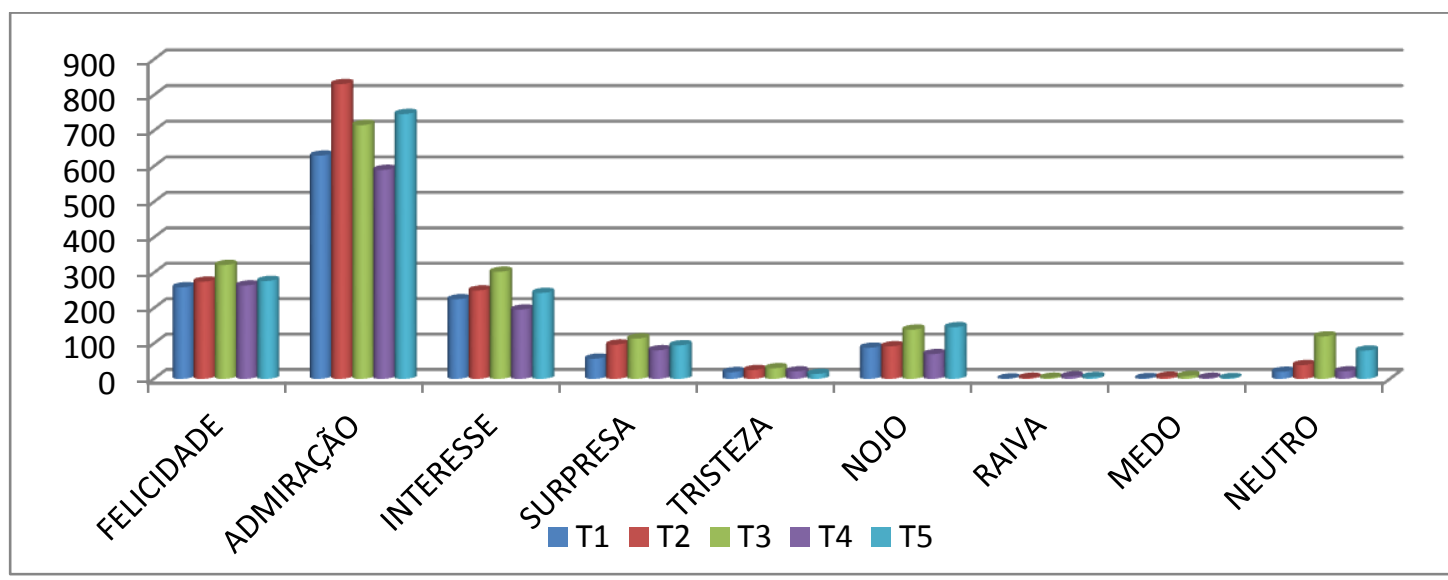

Gráfico 2 - Emoções identificadas nos Chats

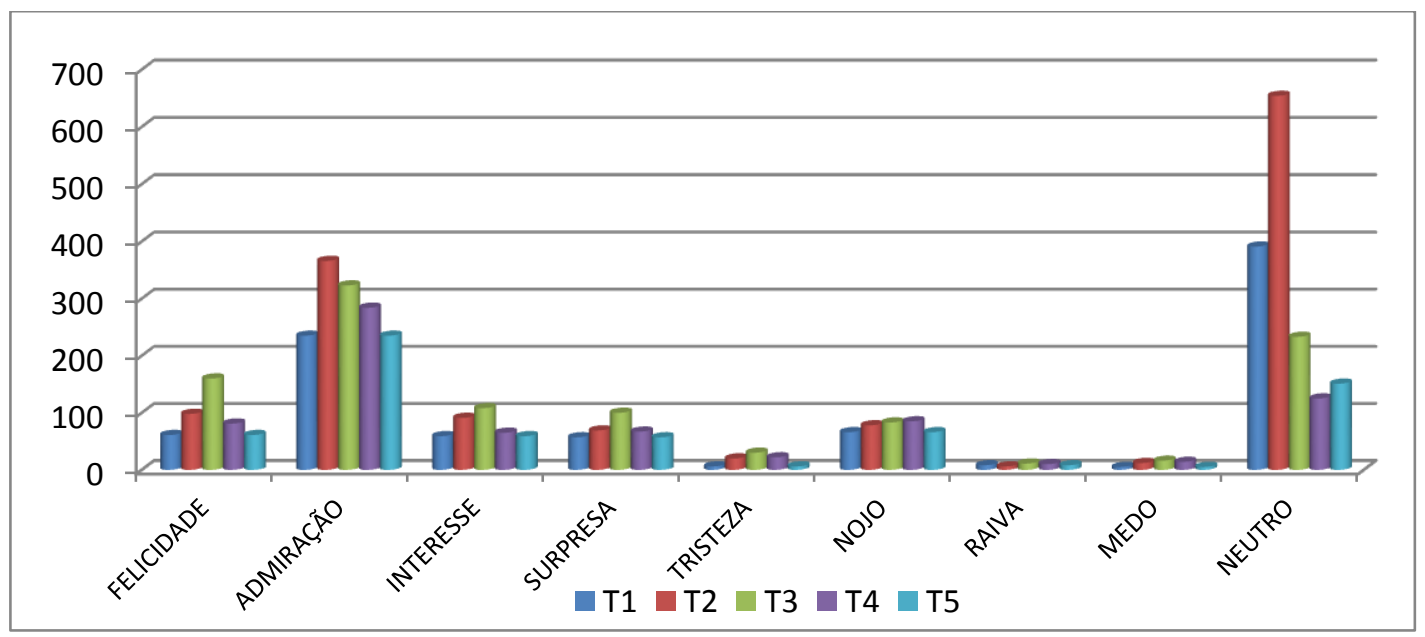

Com os resultados apresentados nos Gráficos 1 e 2, podemos observar a importância da análise de sentimentos nos textos produzidos pelos alunos em cada turma. Com essas informações podemos medir o humor do aluno na realização de cada atividade. Verificamos que na atividade fórum temos 10,81\% mensagens (negativas+neutras) e na atividade chat temos 35,34\% mensagens (negativas+neutras). As informações podem ajudar na análise da turma, considerando que esses valores podem representar insatisfação, dificuldades e desinteresse dos alunos, com isso podemos melhorar esse cenário por meio de estratégias pedagógicas, que aprimorem a atuação do tutor, convertendo muitos desses resultados em positivos.

Com o uso da análise de sentimentos o tutor pode acompanhar o humor de cada aluno no decorrer do curso, servindo como mais uma ferramenta de apoio, tendo em vista o grande número de postagens nos fóruns e chats do AVA. Em geral os textos postados nos fóruns são mais longos e nos chats são mais curtos, essa diferença foi percebida no processamento das mensagens, pois o número de palavras não identificadas nos fóruns foi maior que no chat. 
VI Congresso Brasileiro de Informática na Educação (CBIE 2017)

Anais do XXVIII Simpósio Brasileiro de Informática na Educação (SBIE 2017)

\section{Conclusões e Trabalhos Futuros}

As pesquisas utilizando análise de sentimentos tem se destacado nos últimos anos, principalmente por conta dos avanços tecnológicos, aplicações no campo educacional podem ajudar professores a vislumbrar novas possibilidades de utilizar a emoção como mediadora da aprendizagem.

A partir dos experimentos iniciais realizados neste trabalho, observamos que a ferramenta SenticNet classifica corretamente as palavras, contudo muitas palavras não foram identificadas tendo vista a limitação da base de conhecimento da ferramenta com textos no idioma português do Brasil, dificultando uma análise melhor dos sentimentos expressos pelos alunos

Podemos destacar também a importância da interpretação da emoção manifestada por estudantes no AVA, entender as dimensões afetivas influenciam na aprendizagem tanto positiva quanto negativamente, com isso ter mais um instrumento que possa ajudar o tutor é fundamental.

Os resultados obtidos até o momento são promissores, evidenciando a importância do uso da Computação Afetiva no acompanhamento de alunos durante o processo de ensinoaprendizagem, além de melhorar a qualidade dos cursos.

Como trabalhos futuros pretendemos adicionar novos gestos ao avatar, aperfeiçoar a base de conhecimento, utilizar outras ferramentas para analisar os sentimentos e comparar os resultados obtidos com resultados produzidos por humanos.

\section{Referências}

Alencar, M.A.S. (2011a) Sistema Multiagente para Apoiar a Percepção e o Acompanhamento de Atividades em Ambientes Virtuais de Aprendizagem. Dissertação de Mestrado em Informática, Universidade Federal do Amazonas (UFAM).

Alencar, M.A.S.; Netto, J.F.M. (2011b) Improving Cooperation in Virtual Learning Environments Using Multi-Agent Systems and AIML. In: Frontiers in Education Conference, 41, Rapid City, South Dakota, USA, v.1, p. 1713-1719, 2011.

Alencar, M.A.S.; Netto, J.F.M. TUtor Collaborator Using Multi-Agent system, Collab. Technol. Social Comput., vol. 460, pp. 153-159, 2014.

Amini, R.; Lisetti, C.; Ruiz, G. (2015) HapFACS 3.0: FACS-Based Facial Expression Generator for 3D Speaking Virtual Characters.IEEE Trans. Affective Computing 6(4): 348-360 (2015)

Baldassarri S.; Cerezo E. (2012) Maxine: Embodied Conversational Agents for Multimodal Emotional Communication . Computer Graphics, ISBN: 978-953-51-0455-1, pp.195-211, ed. InTech

Bastos, H.P.P.; Wives, L. K. ; Bercht, M. Presença Social e Pertencimento em Fóruns Educacionais: Manifestação e Percepção de Afetividade. In: XXII Simpósio Brasileiro de Informática na Educação (SBIE), 2011, Aracaju.

Cambria, E., S. Poria, Bajpai, R.; Schuller, W.S. 2016. SenticNet 4: A semantic resource for sentiment analysis based on conceptual primitives. In: COLING, 2666-2677.

Clavel, C.; Callejas, Z. Sentiment Analysis: From Opinion Mining to Human-Agent Interaction. Affective Computing IEEE Transactions on, vol. 7, pp. 74-93, 2016, ISSN 1949-3045. 
VI Congresso Brasileiro de Informática na Educação (CBIE 2017)

Anais do XXVIII Simpósio Brasileiro de Informática na Educação (SBIE 2017)

Cruz, S. R. M.; Souza, A. M. Afetividade e EaD: caminhos possíveis. In: ESUD 2014- XI Congresso Brasileiro de Educação a Distância, 2014, Florianópolis SC.

Ekman, P. (2011). A linguagem das emoções (C. Szlak, Trad.). São Paulo: Lua de Papel. (Obra original publicada 2003).

Fontes, L. M. O. ; Mendes Neto, F. M. ; Diniz, F. A. ; Jácome Junior, L. ; Silva, L. C. N. ; Carlos, D. G. . Um Agente Pedagógico Animado de Apoio à Aprendizagem Baseada em Problema Utilizando o Moodle. In: Workshop sobre Avaliação e Acompanhamento da Aprendizagem em Ambientes Virtuais. Congresso Brasileiro de Informática na Educação (CBIE), 2012, Rio de Janeiro.

Frozza, R.; Silva, A. K.; Lux, B.; Cruz, M.J.K ; Borin, Mirceia. Dóris 3D: Agente Pedagógico baseado em Emoções. In: XX Simpósio Brasileiro de Informática na Educação, 2009, Florianópolis, p. 1-10.

Graesser,A.C.;D’Mello, S.K.;Hu,X.;Cai, Z.;Olney, A.;Morgan, B.(2012). AutoTutor. In P. McCarthy and C. Boonthum-Denecke (Eds.), Applied Natural Language Processing: Identification, Investigation, and Resolution (pp. 169-187). Hershey, PA: IGI Global.

Jaques, P.; Nunes, M. A. S. N. Ambientes Inteligentes de Aprendizagem que inferem, expressam e possuem emoções e personalidade. Jornada de Atualização em Informática na Educação (JAIE). 1 ed. RJ, 2012, v. , p. 31-82.

Liu, B. Opinions, Sentiment, and Emotion in Text. Cambridge University Press, p. 381, 2015.

Longhi, M. T.; Behar, P. A.; Bercht, M. e Simonato, G. Investigando a subjetividade afetiva na comunicação assíncrona de ambientes virtuais de aprendizagem. In: XX Simpósio Brasileiro de Informática na Educação (SBIE), Florianópolis, SC. Novembro, 2009.

Machado, A. A. A. ; Longui, M. T. ; Nunes, M. A. S. N. ; Pardo, T.A.S.. Personalitatem Lexicon: Um Léxico em Português Brasileiro para Mineração de Traços de Personalidade em Textos. Proceedings of the XXVI Simpósio Brasileiro de Informática na Educação, v. 1, p. 1122-1126, Maceió, 2015.

Mateus, G.; Wilges, B.; Diniz, D.; Nassar, S.; Wronscki, V.; Cislaghi, R. An Animated Pedagogical Agent supporting student learning using performance analysis from a Fuzzy Agent. In: XXV Simpósio Brasileiro de Informática na Educação, 2014, Dourados.

Martins, I.; Morais, F.; Schaab, B.; Jaques, P. A. O Impacto dos gestos de Agentes Pedagógicos Animados na compreensão de dicas pelos aprendizes. In: Simpósio Brasileiro de Informática na Educação (SBIE), 2014, Dourados.

Niewiadomski, R.; Pelachaud, C. Affect expression in ECAs: application to politeness displays, International Journal of Human-Computer Studies, Volume 68, Issue 11, November 2010, Pages 851-871.

Olney, A., D'Mello, S., Person, N., Cade, W., Hays, P., Williams, C., Lehman, B., \& Graesser, A. (2012). Guru: A Computer Tutor that Models Expert Human Tutors. Proceedings of the 11th International Conference on Intelligent Tutoring Systems, (pp. 256-261). Berlin: Springer

Picard, R.W. (2003). Affective Computing: Challenges. International Journal of HumanComputer Studies, Volume 59, Issues 1-2, July 2003, pp. 55-64.

Sansonnet, J.; Correa, D.W.; Jaques, P.A ; Braffort, A. ; Verrecchia, C. (2012) Developing Web fully-integrated conversational assistant agents. In: ACM Research in Applied Computa-tion Symposium (RACS), 2012, Texas. p. 14-19 